\title{
Contribución de Centros y Escuelas de Excelencia de Institutos Nacionales de Salud al recurso humano
}

Contribution of Centers and Schools of Excellence of the National Institutes of Health to human resources Contribuição dos Centros e Escolas de Excelência dos Institutos Nacionais de Saúde para os recursos humanos

\section{ARTÍCULO GENERAL}

\section{LLimi Renzo López Cruz}

Universidad de San Martín de Porres, Lima - Perú

\section{Miriam Rosario Salazar Robles}

Universidad de San Martin de Porres, Lima - Perú

Recibido 15 de Febrero 2021 | Arbitrado y aceptado 03 de Marzo 2021 | Publicado el 01 de Junio 2021

\section{RESUMEN}

El objetivo fue identificar la situación actual de las Centros y Escuelas de Excelencia del Perú en los Institutos Nacionales de Salud y su contribución al fortalecimiento del Recurso Humano, buscando identificar elementos que obstaculizan o facilitan su desarrollo y su formalización como Centros y Escuelas de Excelencia, asimismo, permitirá analizar el efecto de las fuerzas de Salud Internacional en el desarrollo y el fortalecimiento de los Centros y Escuelas de Excelencia de los Institutos Nacionales de Salud. Para ello, se propuso una metodología de tipo cualitativo transversal. La técnica fue la recolección de datos a través de la aplicación de la encuesta, entrevista semiestructuradas a los actores claves de los institutos; así como la revisión de documentos nacionales e internacionales. Los resultados señalaron que hay 9 Institutos Nacionales en Salud, de los cuales solo un Instituto Nacional Especializado en Salud cuenta con un Centro y/o Escuelas de Excelencia, lo cual representa el $11 \%$. Si bien es cierto no todos los Institutos cuentan con Centros o escuelas de excelencia, todos desarrollan actividades de fortalecimiento al Recurso Humano según sus funciones teniendo como enfoque el modelo de desarrollo, repercutiendo en una mejor prestación de servicios de salud sin embargo no articulando entre ellos y menos con la Escuela Nacional de Salud Pública. Se concluyó que los Centros y Escuelas de Excelencia de Los Institutos Nacionales en Salud contribuyen al fortalecimiento del recurso humano mediante la docencia, investigación y asesoramiento a nivel nacional e internacional, con diferentes actores (Públicos, Privados, ONGs, etc).

Palabras clave: Centros o Escuelas de Excelencia; Recursos humanos para la salud; Salud Internacional; Salud.

\section{ABSTRACT}

The objective was to identify the current situation of the Centers and Schools of Excellence of Peru in the National Institutes of Health and their contribution to strengthening the Human Resource, seeking to identify elements that hinder or facilitate their development and their formalization as Centers and Schools of Excellence. Likewise, it will make it possible to analyze the effect of the forces of International Health on the development and strengthening of the Centers and Schools of Excellence of the National Institutes of Health. For this, a cross-sectional qualitative methodology was proposed. The technique was the collection of data through the application of the survey, semi-structured interviews with the key actors of the institutes; as well as the review of national and international documents. The results indicated that there are 9 National Institutes of Health, of which only one National Institute Specialized in Health has a Center and/or Schools of Excellence, which represents $11 \%$. Although it is true that not all the Institutes have Centers or schools of excellence, they all develop activities to strengthen Human Resources according to their functions, focusing on the development model, having an impact on a better provision of health services, however, not articulating between them. and less with the National School of Public Health. It was concluded that the Centers and Schools of Excellence of the National Institutes of Health contribute to the strengthening of human resources through teaching, research and advice at a national and international level, with different actors (Public, Private, NGOs, etc).

Keywords: Centers or Schools of Excellence; Human resources for health; International Health; Health.

\section{RESUMO}

O objetivo foi identificar a situação atual dos Centros e Escolas de Excelência do Peru nos Institutos Nacionais de Saúde e sua contribuição para o fortalecimento do Recursos Humanos, buscando identificar elementos que dificultam ou facilitam seu desenvolvimento e sua formalização como Centros e Escolas de Excelência Da mesma forma, permitirá analisar o efeito das forças da Saúde Internacional no desenvolvimento e fortalecimento dos Centros e Escolas de Excelência dos Institutos Nacionais de Saúde. Para isso, foi proposta uma metodologia qualitativa de corte transversal. A técnica foi a coleta de dados por meio da aplicação do survey, entrevistas semiestruturadas com os principais atores dos institutos; bem como a revisão de documentos nacionais e internacionais. Os resultados indicaram que existem 9 Institutos Nacionais de Saúde, dos quais apenas um Instituto Nacional Especializado em Saúde possui um Centro e/ou Escolas de Excelência, o que representa $11 \%$. Embora seja verdade que nem todos os Institutos dispõem de Centros ou Escolas de excelência, todos desenvolvem atividades de reforço dos Recursos Humanos de acordo com as suas funções, apostando no modelo de desenvolvimento, impactando numa melhor prestação de serviços de saúde, no entanto, não articulando entre eles e menos com a Escola Nacional de Saúde Pública. Concluiuse que os Centros e Escolas de Excelência dos Institutos Nacionais de Saúde contribuem para o reforço dos recursos humanos através do ensino, investigação e assessoria a nível nacional e internacional, com diferentes atores (Público, Privado, ONG, etc).

Palavras-chave: Centros ou Escolas de

Excelência; Recursos humanos para a saúde; Saúde Internacional; Saúde. 


\section{Introducción}

El Sistema Sanitario Peruano es sumamente complejo debido a la fragmentación que lo caracteriza, en la medida que está compuesto por subsectores como el Ministerio de Salud (MINSA) que cubre el 60\% de la demanda; EsSalud, que satisface el 30\% y las Fuerzas Armadas (FFAA), la Policía Nacional (PNP) y el sector privado que abarcan el resto. Así, el sistema de salud tiene numerosos proveedores y seguros con coordinaciones deficientes y que a menudo desempeñan funciones superpuestas. Asimismo, el recurso humano suele tener diversos trabajos en cinco subsectores. (1)

Adicional a estos subsectores, otro factor importante en la problemática, es la distribución geográfica desigual del recurso humano, aun cuando se ha reconocido que estos representan el componente fundamental para garantizar una atención de calidad a la población por lo que debería trabajarse de forma integral. (2) Así, existen retos pendientes en materia de mejora, precisamente relacionados a la distribución. (3) En ese sentido, Lima concentra la mayor cantidad de proveedores de salud, mientras que las regiones presentan deficiencias. Entre las más bajas se registran los departamentos de Piura (9.6), Lambayeque (10.7) y Loreto (10.8) (1). Asimismo, la densidad de recursos humanos actual en el país es de 17,8 agentes por cada 10.000 habitantes.

Por ello, en la última década, se ha experimentado un aumento de la demanda de proveedores, especialmente de médicos especialistas, debido a la aplicación de un seguro de salud universal y las políticas asociadas al mismo. (1) En el periodo de 2010 - 2016 se observó un incremento de médicos especialistas en 43\%. (4) Sin embargo, están concentrados en un mayor porcentaje en la capital habiendo un deficit en las regiones.

Por otro lado, la complejidad del problema sanitario, los problemas de salud descritos párrafos arribas, así como los nuevos retos que enfrenta el sistema sanitario hacen que sea necesario actuar de forma proactiva para fortalecer las capacidades del recurso humano de manera permanente, creativa, con uso de la tecnologias de manera coordinada con las regiones del país.

En el país estos procesos de fortalecimiento de competencias y capacidades del recurso humano en salud tanto técnica operativa como académica se ha centrado en las Instituciones educativas publicas y privadas orientandose a la transparencia del mercado 
laboral; el MINSA a traves de la Escuela Nacional de Salud Publica la cual se encuentra en proceso de reactivación, cuenta como propuesta el construir programas de estudios estructurando procesos de enseñanza y aprendizaje que esten acorde con las políticas y las demandas de las problemas sanitarios de la población.

Por otro lado, en la estructura del ápice del sistema de los servicios de salud se encuentran los 8 Institutos Especializados en Salud y 1 Instituto Nacional de Salud Pública, en adelante "Institutos" cuyos roles se basan en el tratamiento de alta especializacion, educación e investigación, formación de especialistas a nivel nacional, promover capacitaciones o entrenamientos a otros profesionales de la salud en los diferentes niveles de atención a través de los Centros y/o Escuelas de Excelencias, como estrategia de intervención.

Esta estrategia propuesta generará, la necesidad de implementar un proceso colaborativo entre los mencionados "Institutos" y la Escuela Nacional de Salud Pública para fortalecer el sistema de salud estableciendo mecanismos de integración y formación de competencias del recurso humano para el cumplimiento de las políticas sectoriales, lo cual permitirá disminuir las brechas del acceso a los conocimientos basado en evidencias de manera descentralizadas impulsando las nuevas tecnológicas para mejorar las respuestas a las necesidades de los usuarios por medio de la transferencia de conocimiento. Los cuáles serán medidos a través de los números de procesos colaborativos, mecanismo de cooperación nacional e internacional, numero de recursos, capacidades con las que cuentan, metodologías de trabajo, etc.

Con ello planteado, se indica que el objetivo general de la presente fue Identificar el estado actual de los Centros y Escuelas de Excelencia de los Institutos Nacionales de Salud del Perú para el fortalecimiento del recurso humano en salud. Asimismo, se tuvieron objetivos específicos como: Mapear los Centros y/o Escuelas de Excelencia en los Institutos Nacionales de Salud en el Perú; Identificar la contribución de los Centros y/o Escuelas de Excelencia de los Institutos Nacionales de Salud al fortalecimiento del recurso humano en el país teniendo como enfoque el modelo de desarrollo; Analizar el efecto de las fuerzas de Salud Internacional en el desarrollo y el fortalecimiento de los Centros y Escuelas de Excelencia de los Institutos Nacionales de Salud para el fortalecimiento del recurso humano. 


\section{Materiales y métodos}

Se tuvo como diseño y tipo de Investigación, un estudio caso, cualitativo descriptivo y transversal. Las técnicas que se utilizaron para la recolección de información fueron: reuniones con los directores/representantes de los Institutos Nacionales de salud, entrevistas a los coordinadores de los Centros/Escuelas de Excelencia ya fin de llegar a determinar recoger información que permita cumplir los objetivos planteados tanto general como específicos propuestos.

La Entrevista semiestructurada: Se realizó a partir de una guía prediseñada que contuvo las preguntas formuladas a expertos previamente seleccionados de acuerdo a criterios, con la finalidad de obtener la información requerida.

Revisión bibliográfica y documental: La técnica de revisión bibliográfica sistemática, fue utilizada para recopilar información de los diversos documentos nacionales e internacionales que abordan la temática. Asimismo, los miembros de la comunidad de práctica aportaron documentos y referencias que contribuyeron para la elaboración del proyecto y análisis de resultados.

Asimismo, se planteó una intervención. La población a intervenir fueron los actores claves de los centros y/ escuelas de excelencia de los Institutos Nacionales de Salud. El objeto de intervención fue la identificación de los Centros y Escuelas de Excelencia en Salud Pública con la cual cuentan los Institutos Nacionales de Salud que fortalecen las capacidades o competencias del recurso humano en Salud.

Se tuvieron como criterios de inclusión Institutos Nacionales de Salud en Lima que participen de forma voluntaria; y de exclusión: Aquellos que no deseen participar.

El modo de evaluación buscó que el proyecto cumpliera con el objetivo propuesto basado en la metodología que contribuya en la identificación del estado actual de los Centros y Escuelas de Excelencia, que desarrollen y fortalezcan las capacidades y competencias del Recurso Humano en salud. Dicha información se basa en los siguientes puntos:

- $\quad$ Revisión de documentos técnicos y normativos

- $\quad$ Establecimiento de Objetivos, metas e indicadores 
- $\quad$ Establecer una evaluación basada en resultados concretos

- $\quad$ Articulación de los Institutos Nacionales en Salud

- $\quad$ Análisis de datos y estrategias para el fortalecimiento del talento humano y la ENSAP.

Los indicadores de evaluación fueron los obtenidos en base a los resultados para ver el cambio de mejoras e implementación en los procesos de consolidación de los centros y escuelas de excelencia.

Entre los Procedimientos y técnicas para sistematizar la información, se tuvo las encuestas, los instrumentos de recolección de datos y las entrevistas semiestructuradas aplicadas las cuales fueron evaluados en las áreas de centros o escuelas de excelencia o sus simili, y en el personal que labora en dichas áreas.

El análisis de datos y los procesos de evaluación fueron realizado a través del programa SPSS y Excel.

Entre las estrategias para asegurar la adherencia, se realizaron talleres de sensibilización, reuniones de socialización y contacto a frecuente través de diferentes redes sociales. Entre una de las intervenciones concomitantes realizadas tenemos a la realizada en el marco del fortalecimiento de la cooperación internacional en dichas instituciones a través de la reactivación de las redes de cooperación.

\section{Aspectos éticos}

El trabajo fue realizado tomando en consideración los principios de transparencia reflejando este aspecto en la accesibilidad y recojo de información pública de entidades internacionales y también aquellas pertenecientes al estado peruano. Asimismo, se llevó a cabo la redacción del artículo respetando las normas de citación del formato Vancouver en la medida que toda información ajena fue citada y referenciada correcta $\mathrm{y}$ oportunamente en el documento, pensando también en el lector a quien se le pudiera facilitar la búsqueda de las fuentes con veracidad.

\section{Resultados}


Entre los resultados obtenidos se tiene la relación de correspondencia con los objetivos propuestos:

- $\quad$ Primer Objetivo Específico: Mapear los Centros y/o Escuelas de Excelencia en los Institutos Nacionales de Salud en el Perú

\section{Respecto a la identificación de los Institutos}

Se identificaron que existen nueve institutos nacionales de salud, lo cual representan el 100\% de la muestra. Del total de institutos, ocho son considerados como especializados, los cuales representan el $89 \%$ del total, y un instituto considerado como de Salud Pública, el cual representa el 11\% del total. Asimismo, del total de Institutos encontrados, ocho se encuentran registrados como Instituciones Prestadoras de Servicios de Salud (IPRESS), los cuales representan el 89\% del total, y un instituto (INS) no se encuentra registrado como IPRESS, el cual representa el 11\% del total.

\section{Respecto a Centros de referencia}

Respecto al análisis de los institutos como centros de referencia se encontró que el $100 \%$ de los Institutos se considera centro de referencia a nivel nacional y solo el $67 \%$ se considera centro de referencia internacional. Asimismo, el $89 \%$ de los institutos son centro de referencia a nivel nacional y solo el 33\% son centro de referencia internacional, de acuerdo a su acreditación y representación en representación del MINSA a nivel nacional e internacional.

En cuanto a su estructura organica en docencia e investigación, 56\% sí está en una estructura orgánica y el $44 \%$ es de manera funcional adscrita a una dirección de línea

\section{Respecto a los instrumentos de gestión}

Con respecto a los instrumentos de gestión, el 85\% sí cuenta con (PEI, POI, MAPRO, etc.), un 44\% en instrumentos educativos (normas, directivas, reglamentos, etc.).

\section{Respecto a la gestión del presupuesto}

Con respecto a la gestión del presupuesto, el 89\% (es decir 8 de 9 Institutos) cuenta con una asignación prespuestal para docencia e insvestigacion siendo el Instituto Nacional de Salud el que cuento con mayor presupuesto (Figura 1) 


\section{Respecto a Centros/Escuela de Excelencia}

El 11\% de institutos (es decir 1 de 9 Institutos) cuentan con centro/ escuela de excelencia educativa a nivel nacional e internacional, siendo el Instituto Nacional de Enfermedades Neoplásicas que cuenta con ambos. Asimismo, dicha institución cuenta con coordinadores designados para la gestión de los mismos.

\section{Respecto a la Prestación de Servicios Educativos}

Se aprecia que los institutos prestan una serie de servicios educativos que deben de ser conocidas para ser aprovechados de forma más eficiente (Figura 2).

\section{Respecto a la Gestión de los Servicios Educativos}

Se aprecia que los institutos prestan una serie de mecanismo de gestión de los servicios educativos que deben de ser difundidas y trabajas de manera más colaborativa.

Segundo Objetivo Específico: Identificar la contribución de los Centros y/o Escuelas de Excelencia de los Institutos Nacionales de Salud al fortalecimiento del recurso humano en el país teniendo como enfoque el modelo de desarrollo.

Es muy importante manifestar que para que los institutos fortalezcan al recurso humano en el país de acuerdo al enfoque de modelo de desarrollo es necesario conocer los siguientes resultados que están relacionados a este segundo objetivo tales como:

\section{Respecto a la estructura del contenido de Capacitación}

El $100 \%$ si realiza capacitaciones, y de formación de profesionales de la salud pregrado y postgrado. Con respecto al tipo de capacitación tenemos que el $67 \%$ en innovación y desarrollo, $78 \%$ en gestión, $100 \%$ en diagnóstico y tratamiento, $89 \%$ en prevención secundaria y terciaria; y $78 \%$ en prevención primaria.

\section{Respecto a las metodologías educativas}

Con respecto a la metodologias educativas que fortalezcan al recurso humano el $68 \%$ de los institutos aplica algun tipo de metodologia de capacitacion como presencial, semipresencial o virtual; siendo que el 100\% de los Institutos sí cuentan con metodologías virtuales a nivel nacional (Figura 4).

\section{Respecto al Recurso Humano}


Respecto al Recurso Humano para realizar la capacitación, se encontró que en los institutos Especializados el 76\% del recurso humano son especialistas, asimismo 11\% tiene grado de magister, el 2\% de doctor y $11 \%$ de subespecilidades. Asimismo, si cuentan con docentes, tutores, facilitadores tanto nacionales y algunos internacionales. Esto nos explica que si existe competencias con alta especilidad para transmitir los conocimientos y así fortalecer al recurso humano del pais.

\section{Respecto a la Tecnología educativa}

Con respecto a la tecnologia educativa, el $67 \%$ aplica alguna tecnologia educativa. El 89\% aplica Telesalud y a traves de un aula virtual (Figura 5).

Tercer Objetivo Específico: Analizar el efecto de las fuerzas de Salud Internacional en el desarrollo y el fortalecimiento de los Centros y Escuelas de Excelencia de los Institutos Nacionales de Salud para el fortalecimiento del recurso humano

\section{Respecto a las Alianzas estratégicas en cooperación}

Con respecto al Efecto de las relaciones internacionales a través de las alianzas estratégicas en cooperación tenemos que a nivel nacional los institutos presentan 100\% de alianzas con instituciones públicas y privadas. Caso contrario con respecto a nivel internacional donde las alianzas a nivel privado representan el $67 \%$ y a nivel público solo el $56 \%$.

\section{Respecto a Redes de Gestión}

Con respecto al Efecto de las relaciones internacionales a través de las redes de gestión en los institutos a nivel nacional es de $78 \%$ y a nivel internacional es de $56 \%$.

\section{Respecto a la Participación en la OMS}

Con respecto al Efecto de las relaciones internacionales a través de la participación de los institutos en la OMS, tenemos que el 22\% contribuye en la preparación a los programas de la OPS/OMS, el 11\% contribuye en competencias de los programas de la OPS/OMS y el 22\% contribuye en capacidades a los programas de la OPS/OMS.

\section{Discusión}

De los hallazgos, se tuvo en primer lugar la identificación de los institutos nacionales de salud, de los cuales se identificó ocho en el país. Esta información es 
complementaria con lo indicado por el Ministerio de Salud que señalaron a los INS asociándolos con el objetivo de proponer políticas y normas, promover, desarrollar y difundir la investigación científica-tecnológica y brindar servicios de salud en los campos de salud pública, control de enfermedades transmisibles y no transmisibles, alimentación y nutrición, producción de biológicos, control de calidad de alimentos, productos farmacéuticos y afines, salud ocupacional, protección del medio ambiente y salud intercultural, para contribuir a mejorar la calidad de vida de la población. (9)

Asimismo, se indicó en la presente respecto al análisis de los institutos como centros de referencia, que el 100\% de los Institutos se considera centro de referencia a nivel nacional y solo el $67 \%$ se considera centro de referencia internacional. Ello guarda relación con lo mencionado por Vega y Salinas quienes dijeron que uno de los principales desafíos para innovar en la gestión pública a nivel sudamericano guarda relación con crear y gestionar conocimiento de calidad respecto de los asuntos públicos; rol que debiese ser asumido en los centros de referencia de los distintos países, quienes deberían establecer mecanismos de mejora de su papel, desde una base de comparación con los países de referencia a nivel internacional. (10)

También, respecto a los instrumentos de gestión, en Perú pueden especificarse algunos como: Convenios; ROF; Presupuesto Analítico de Personal; Organigrama INS; Manual de Organización y Funciones; Manual de Clasificador de Cargos; Cuadro de Asignación de Personal - CAP; Manual de Procedimientos - MAPRO; Tupa; Políticas Nacionales Evaluación de Indicadores de Desempeño; Reglamento Interno de los Servidores del INS - RIS.

Sobre la gestión y prestación de servicios educativos, uno de los más mencionados fue la telemedicina. Acorde con ello, la Revista Peruana de Medicina Experimental y Salud Pública (RPMESP) dedicó por primera vez en 2015 un número a la informática biomédica y la telesalud alegando que las TIC son herramientas que favorecen la calidad, seguridad, eficiencia y productividad de los servicios de salud. (11)

En cuanto a capacitaciones y metodologías educativas, es importante precisar que, del total del presupuesto asignado, alrededor de la mitad se dirige a entrenamiento y capacitación del personal de salud del INS y de la red de laboratorios del MINSA. (12) Ello debe asociarse igualmente a la tecnología educativa empleada en tales procesos de 
enseñanza y capacitación, un claro ejemplo de ello pueden ser los cursos masivos abiertos en línea (MOOC, por sus siglas en inglés) los que en los últimos años, se han convertido en una herramienta educativa útil para transmitir conocimiento en distintas áreas del saber, cuyas fortalezas (no estar limitados a horarios, idioma o ubicación geográfica) permiten que se realicen desde cualquier dispositivo con acceso a internet. (13)

Por todo lo anterior se indican algunas conclusiones:

- $\quad$ Los INS cumplen con el propósito principal de su creación que es la investigación y el conocimiento, las funciones de educación, servicio y capacitación, en el campo de la salud y formación de personal

Los INS cuentan con una serie de recursos y capacidades para cumplir con sus funciones, pero trabajan de forma desarticulada y sin coordinación.

- $\quad$ Los elementos desde el MCSI que obstaculizan el desarrollo y formalización de los Centros y Escuelas de Excelencia de los INS son la desorganización del sistema, el comercio, los conflictos e intereses; y entre los que facilitan tenemos a la reactivación de la ENSAP, los diversos mecanismos de cooperación y las relaciones internacionales.

Los Centros y Escuelas de Excelencia de los INS contribuyen al fortalecimiento del recurso humano a través de la docencia, investigación y asesoramiento a nivel nacional e internacional, con diferentes actores (Públicos, Privados, ONGs, etc)

\section{Referencias bibliográficas}

Global Health Workforce Alliance. El Perú, s.f. [Internet]. Ginebra: Organización Mundial de la Salud; 2021. Disponible en: http://www.who.int/workforcealliance/countries/per/es/

Inga-Berrospi F, Arosquipa Rodríguez C. Avances en el desarrollo de los recursos humanos en salud en el Perú y su importancia en la calidad de atención. Rev Peru Med $\begin{array}{llll}\text { Exp Salud } & \text { Publica. } & \text { 2019;36(2):312-8. }\end{array}$ http://dx.doi.org/10.17843/rpmesp.2019.362.4493 
Lazo-Gonzales O, Santivañez-Pimentel A. Atención de salud con calidad: desafíos. Lima: Consejo Regional III Lima, Colegio Médico del Perú, 2018.

MINSA. Dirección General de Personal de Salud Observatorio de Recursos Humanos en Salud. Compendio Estadístico: Información de Recursos Humanos del Sector Salud, Perú 2010 -2016. Lima- Perú.

PANLAR. Liga Panamericana de Asociaciones de Reumatología. Proceso de acreditación de centros de excelencia en artritis reumatoide - grupo especial realPANLAR, 2018. Documento técnico PANLAR, 2021. Disponible en: www.panlar.org/sites/default/files/documento_tecnico_espanol.pdf

(http:who.int/collaboratingcentres/es/).

Organización Panamericana de la Salud. Estrategia de Recursos Humanos para el acceso universal a la salud y la cobertura universal de salud. (Internet). 29. a Conferencia Sanitaria Panamericana. 69.a Sesión del Comité Regional de la OMS para las Américas: Del 25 al 29 de septiembre del 2017. Washington DC. OPS: 2017 (Documento CSP 29/10). [Internet]. [citado 7 de enero de 2018]. Disponible en: http://www.paho.org/hq/index.php?option=com_content\&view=article\&id=13497\%3A 29th-pan-american-sanitary-conference \&catid=947\%3Apan-american-sanitary$\underline{\text { conference } \& \text { Itemid }=2105 \& \text { lang }=\text { es }}$

OPS. Abordaje de temas de salud para la perspectiva de salud internacional y la construcción de un modelo conceptual versión 2015. Programa de Líderes en Salud Internacional Edmundo Granda Ugalde (PLSI). Unidad de Recursos Humanos para la Salud (HR). Departamento de Sistemas y Servicios de Salud (HSS). Washington, D.C., 2015. [Internet]. [citado 7 de enero de 2018]. Disponible en: https://cursospaises.campusvirtualsp.org/pluginfile.php/21578/mod_folder/content/0/Sal ud_Internacional_OPS.pdf?forcedownload=1.

Instituto Nacional de Salud INS. Quiénes Somos, s.f. [Internet]. Lima: Ministerio de Salud. Disponible en: https://web.ins.gob.pe/es/acerca-del-ins/informacion-general

Vega A, Salinas C. Análisis de la producción científica en asuntos públicos de Chile y Perú. Desafíos para una mejor gestión pública. LEX - Revista de la facultad de derecho y ciencia política. 2017;15(20). DOI: http://dx.doi.org/10.21503/lex.v15i20.1451 
Curioso WH. La Telesalud y las nuevas fronteras de la informática biomédica en el Perú [editorial]. Rev Peru Med Exp Salud Publica. 2015;32(2):217-20.

Yagui M, Espinoza M, Caballero P, Castilla T, Garro G, Yamaguchi P, et al. Avances y retos en la construcción del sistema nacional de investigación en salud en el Perú. Rev. Perú. Med Exp Salud Pública. 2010; 27(3), 387-397.

Quijano-Escate R, Rebatta-Acuña A, Garayar-Peceros H, Gutierrez-Flores KE, Bendezu-Quispe G. Aprendizaje en tiempos de aislamiento social: cursos masivos abiertos en línea sobre la COVID-19. Rev Peru Med Exp Salud Publica. 2020;37(2):3757. doi: https://doi.org/10.17843/rpmesp.2020.372.5478

\section{Figuras}

Figura 1. Gestión del presupuesto

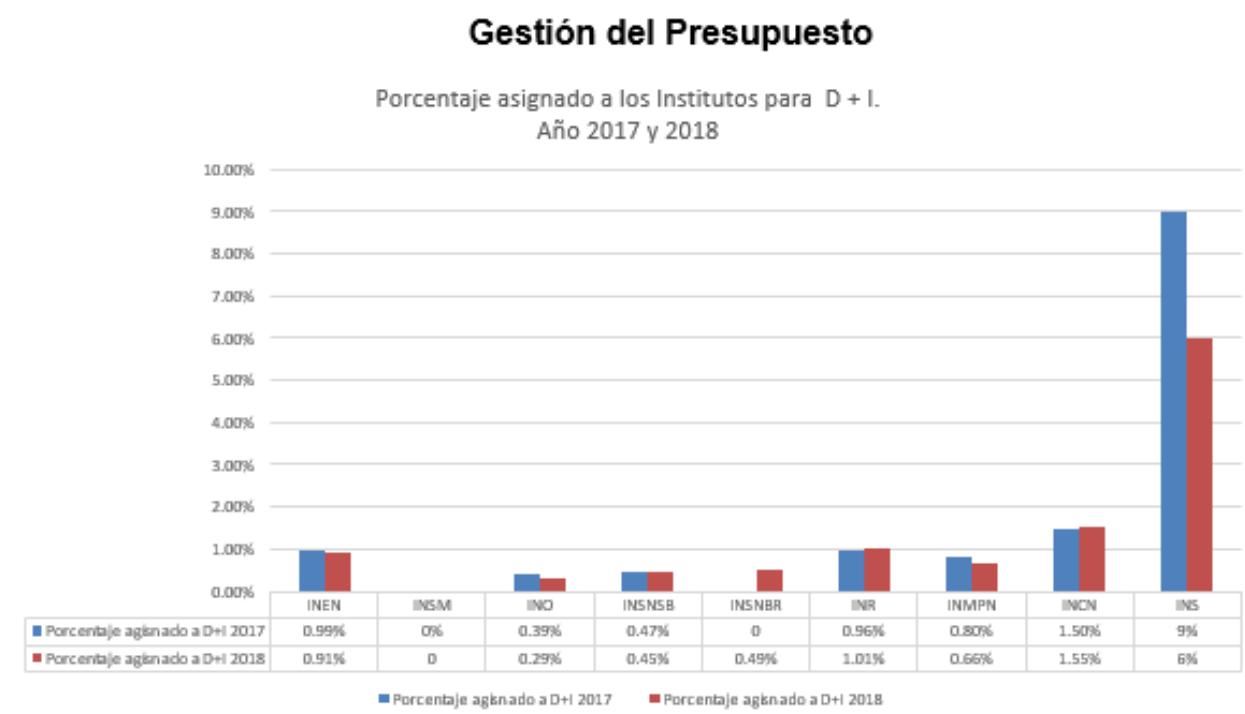

Fuente: Elaboración propia

Figura 2. Prestación de Servicios Educativos 


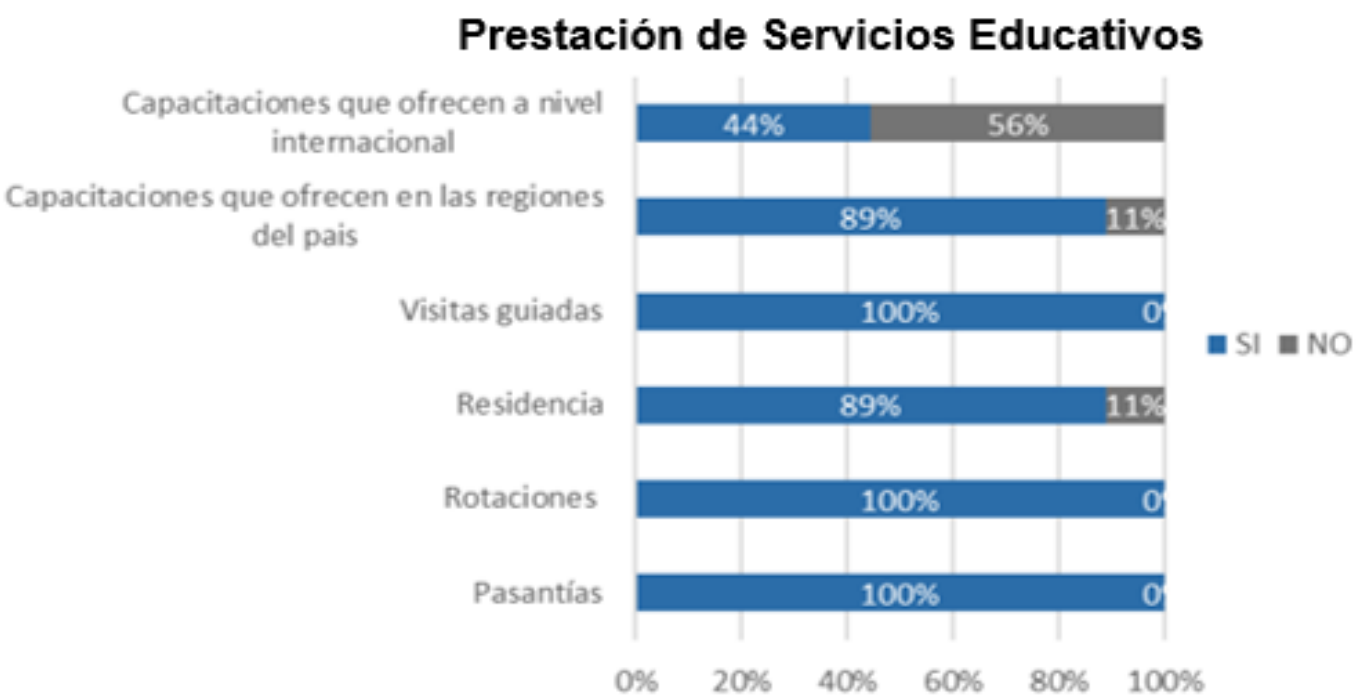

Fuente: Elaboración propia

Figura 3. Gestión de los Servicios Educativos

\section{Gestión de los Servicios Educativos}

Tipos de gestión educativa

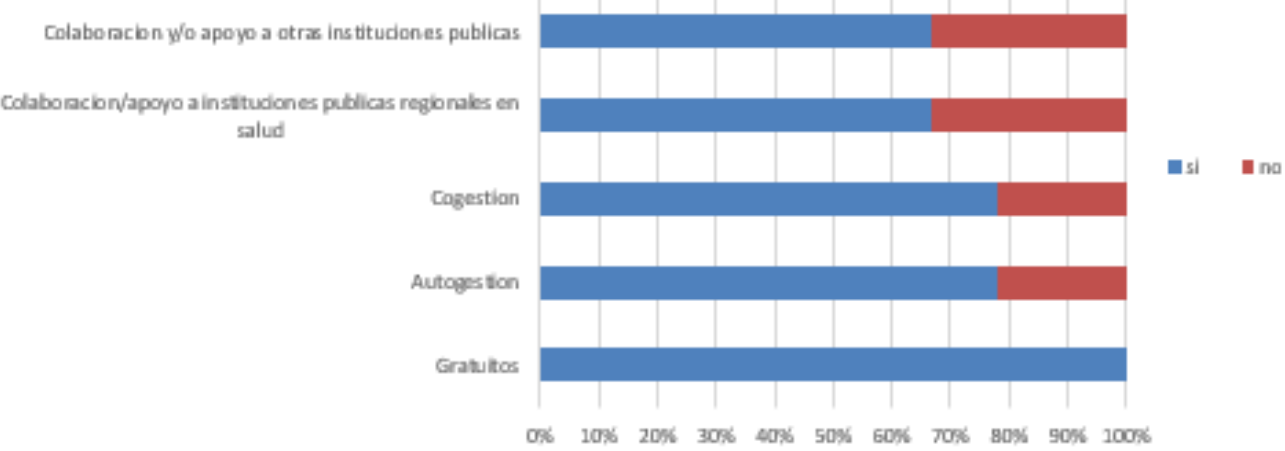

Fuente: Elaboración propia

Figura 4. Metodologías educativas 


\section{Metodología educativa}

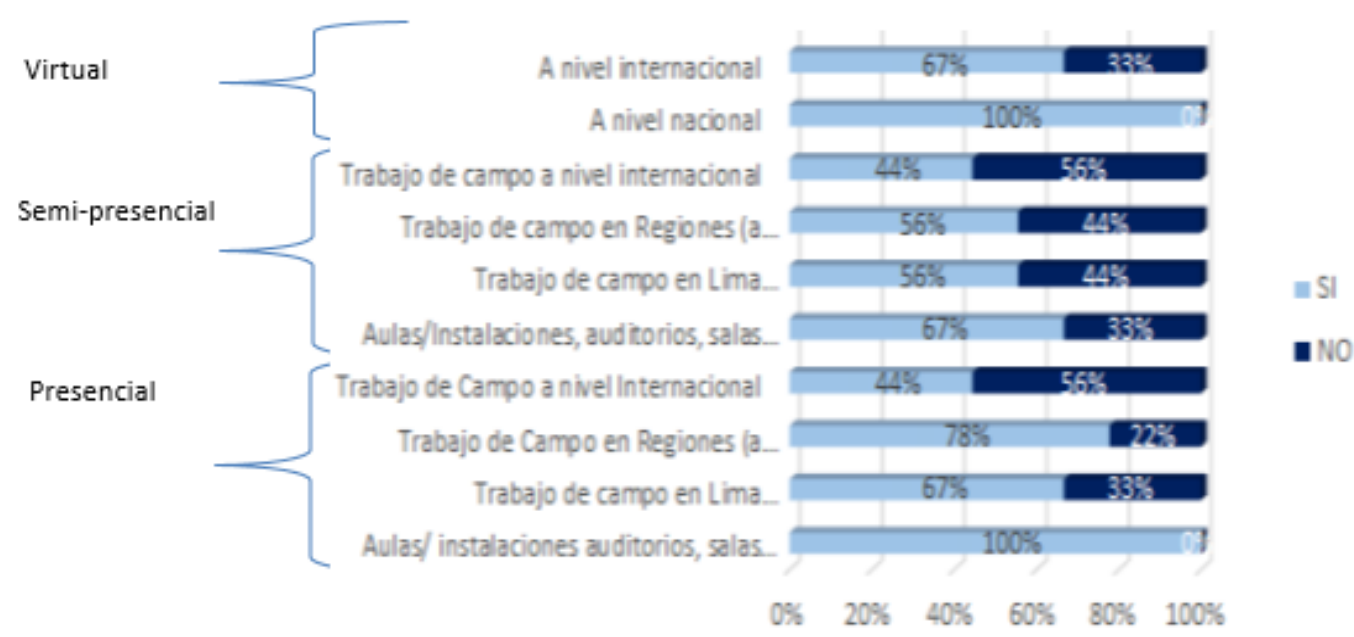

Fuente: Elaboración propia

Figura 5. Tecnología educativa

\section{Tecnología educativa}

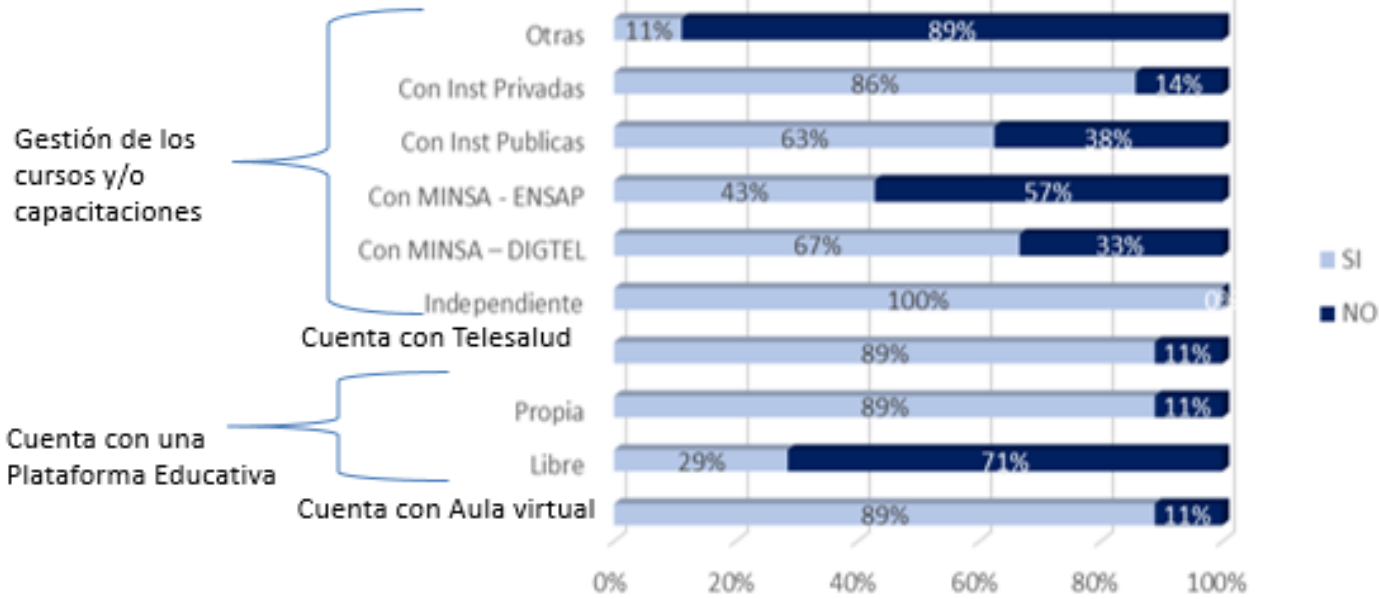

Fuente: Elaboración propia 